\title{
Assigning phrase breaks from part-of-speech sequences
}

\author{
Paul Taylor and Alan W. Black \\ Centre for Speech Technology Research, University of Edinburgh, Edinburgh, UK.
}

\begin{abstract}
This paper presents an algorithm for automatically assigning phrase breaks to unrestricted text for use in a text-to-speech synthesizer. Text is first converted into a sequence of part-of-speech tags. Next a Markov model is used to give the most likely sequence of phrase breaks for the input part-of-speech tags. In the Markov model, states represent types of phrase break and the transitions between states represent the likelihoods of sequences of phrase types occurring. The paper reports a variety of experiments investigating part-of-speech tag-sets, Markov model structure and smoothing. The best setup correctly identifies $79 \%$ of breaks in the test corpus.

(C) 1998 Academic Press Limited
\end{abstract}

\section{Introduction}

An important problem in text-to-speech (TTS) synthesis is to find suitable places in the text for the placement of prosodic phrase breaks. In a typical TTS system, phrase breaks are used by a number of modules, including:

(1) Fundamental frequency contour generation: major phrase boundaries delimit intonation phrases and are the only position where boundary tones can occur. Correct phrasing facilitates suitable accentuation as the last accent in a phrase is treated as the nuclear accent.

(2) Duration: the duration module lengthens segments that occur immediately prior to a phrase boundary.

(3) Pause insertion: pauses can be inserted in the middle of a sentence. The main deciding factor in this is whether a major phrase break has just occurred.

The performance of these modules is heavily dependent on the ability of the phrase break component to place its boundaries in appropriate places.

Past reviews (Ostendorf and Veilleux, 1994) (Wang and Hirschberg, 1992) describe two approaches. The first makes use of the fact that prosodic structure and syntactic structure are related, and uses some sort of syntactic information to predict prosodic

email: paul.taylor@ed.ac.uk; awb@cstr.ed.ac.uk 
boundaries (often in the form of heuristic rules). This approach has several disadvantages that make its use unattractive for real TTS systems. Rule-driven parsers are notoriously unreliable and can provide poor input to the syntax-to-prosody module. In addition, a rule-driven syntax-to-prosody module suffers from the same disadvantages as all rule driven systems: they are often difficult to write, modify, maintain and adapt to new domains and languages.

In light of these shortcomings, some researchers have tried a second approach whereby prosodic structure is derived from robust, if crude, features of the input text. The simplest of these is based on the content word/function word rule (e.g. Silverman 1987) whereby a phrase break is placed before every function word that follows a content word. Despite its simplicity, such an approach can sometimes produce reasonable results. A number of other proposals based on either rule driven or statistical superficial analysis of the text also has been proposed (Veilleux, Ostendorf, Price and Hufnagel, 1990; Wang and Hirschberg, 1992; Hirschberg and Preito, 1994; Ostendorf and Veilleux, 1994).

This paper describes an algorithm of the second type, which assigns phrase breaks using global optimization techniques on sequences of part-of-speech (POS) tags.

\section{Overview of the algorithm}

We define our problem as follows: the input text consists of a sequence of words and between each pair of words is a word juncture. There is a set of $T$ word juncture types and it is the task of a phrase-break algorithm to assign the most appropriate type to each juncture. Most experiments in this paper use two types of juncture, break and non-break. In principle any number of types is possible, for example splitting the break type into minor and major gives three types, or following the ToBI scheme gives five types (Silverman et al., 1992). Here we give an overview of the standard version of our algorithm, which assigns breaks and non-breaks to arbitrary input text.

The algorithm is trained and tested on a database of spoken English in which the original text has been hand annotated with a break label between words that are perceived as being phrase-breaks. The text is tagged with a hidden Markov model (HMM) part-of-speech (POS) tagger that replaces each word by its POS tag. The tags, $c$ are chosen from a tagset $V v_{1}, \ldots, v_{k}$ of size $K$. The juncture between every pair of words is then marked as one of the word juncture types: in the standard case this is either break or non-break.

The algorithm uses a Markov model in which states represent juncture types, and the transitions between states represent the likelihood of particular sequences of breaks and non-breaks occurring. Each state has an observation probability distribution giving how likely that state is to have produced a sequence of part of speech tags. The state observation probabilities are called the POS sequence model and the set of transition probabilities is called the phrase break model. Bayes equation is used to relate the two, and the most likely juncture sequence for a given input can be found by searching through the model and picking the most likely path.

Training the model involves estimating the POS sequence model (the observation probabilities) and the phrase break model (the transition probabilities). 


\subsection{POS sequence model}

The POS sequence model is trained by searching the training data for each juncture type and counting the number of distinct sequences of POS tags before and after the juncture. Generally, the POS sequence is a window of $L$ tags around a juncture $j_{i}, M$ tags preceding $j_{i}$ and $L-M$ tags following $j_{i}$. In our standard system there are two tags before and one after the juncture $(L=3, M=2)$. These counts are converted into probabilities by dividing each count by the total number of occurrences of that juncture type in the data. This gives an estimate of the probability of a POS sequence given a juncture type.

Let us denote a POS sequence $c_{i-M}, . ., c_{i}, . ., c_{i+L-M}$ as $C$ and the number of times this occurs in the training set as $\operatorname{count}(C)$. The number of times a juncture type occurs is given by $\operatorname{count}(j)$. Thus an estimation of the probability is given by:

$$
\tilde{P}(C \mid j)=\frac{\operatorname{count}(C \mid j)}{\operatorname{count}(j)}
$$

which in expanded form is:

$$
\tilde{P}\left(c_{k-M}, \ldots, c_{i-1}, c_{i}, c_{i+1}, \ldots, c_{i+L-M} \mid j_{i}\right)=\frac{\operatorname{count}\left(c_{i-M}, \ldots, c_{i-1}, c_{i}, c_{i+1}, \ldots, c_{i+L-M} \mid j_{i}\right)}{\operatorname{count}\left(j_{i}\right)}
$$

\subsection{The phrase break model}

The phrase break model is trained by examining the database again, but this time ignoring the POS information and only examining junctures. A n-gram of order $N$ is constructed which represents the probability of different sequences of junctures. Using $J_{i-}^{N}{ }^{1}$ to represent the previous sequence of $N$ junctures, we have:

$$
P\left(j_{i} \mid J_{i-1}^{N}\right)=P\left(j_{i} \mid j_{i-1}, j_{i-2}, j_{i-3}, \ldots, j_{i-N+1}\right)
$$

\subsection{Combining the methods}

A network of $T^{N-1}$ nodes and $T^{N}$ arcs is constructed $(N=1$ is a special case and has the same topology as $N=2$ - see Fig. 1). Each node represents a juncture type, and when $N>2$ the nodes represent a juncture in the context of previous junctures. The POS sequence probabilities do not take account of context, and so for a given juncture type are the same no matter where the node occurs in the network. For example, if $N=3$, we will have two break nodes, one for when the previous juncture was a break and one for when the previous juncture was a non-break. These nodes have the same observation probabilities. Figure 1 shows networks for $N=1, N=2$ and $N=3$.

Under this formulation we have the likelihood $P\left(C_{i} \mid j_{i}\right)$ (the POS sequence model) representing the relationship between tags and juncture types, and $P\left(j_{i} \mid j_{i-1}, \ldots, j_{i-N+1}\right)$ (the n-gram phrase break model) which represents the a priori probability of a sequence of juncture types occurring. This is used to give a basic regularity to the break placement, enforcing the notion that phrase breaks are not simply a consequence of local word information. 

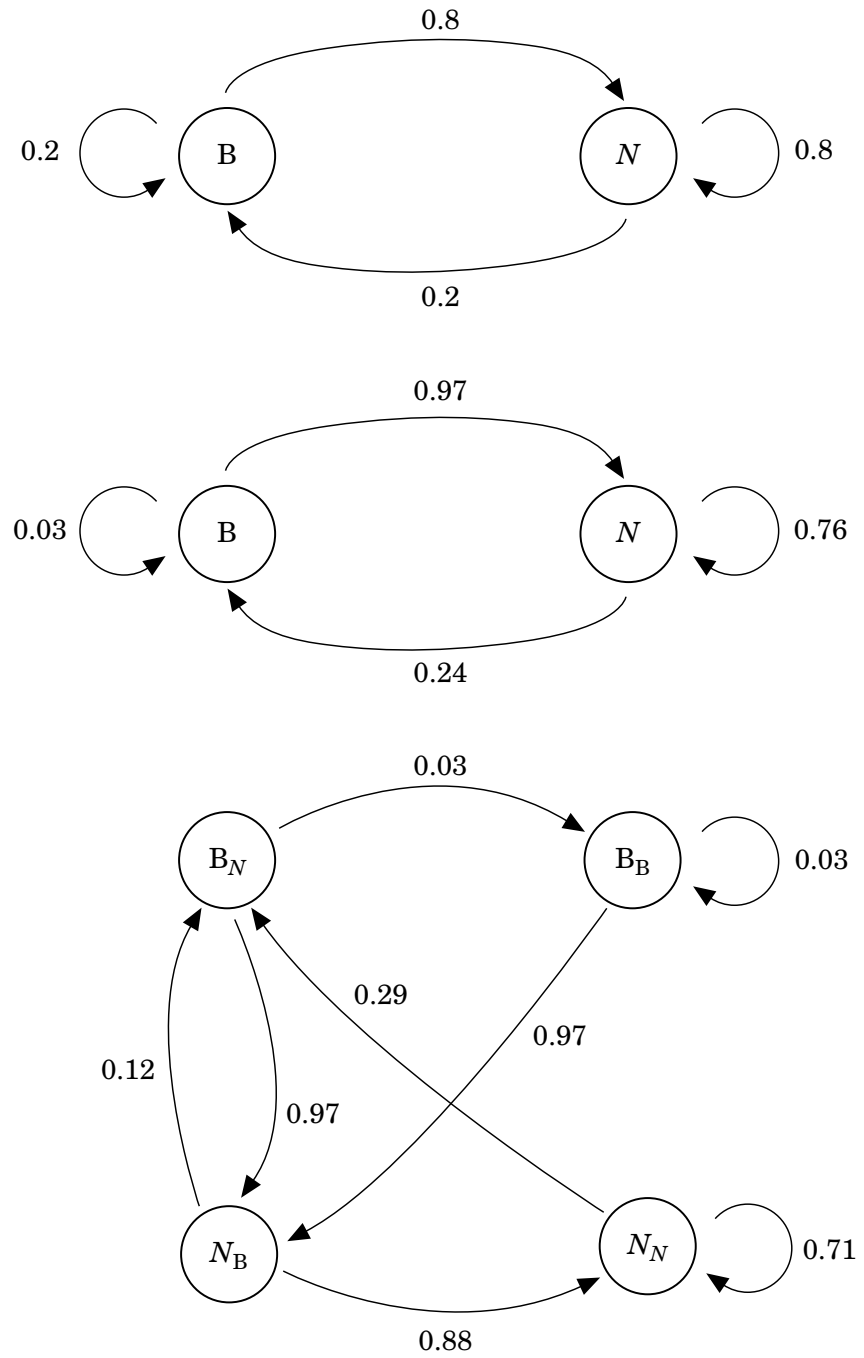

Figure 1. Models for $N=1, N=2$ and $N=3$, showing actual transition probabilities calculated from the training data. The states marked $B$ are for breaks and those marked $N$ are for non-breaks. Subscripts in state names indicate the juncture type of the previous state. In the $N=1$ case the transition probabilities are just the context independent probabilities of the juncture types occurring, i.e. the transition probabilities to a state don't depend on the previous state. In the $N=2$ case, the transition probabilities take into account the previous juncture. Thus in this model it is very unlikely that a break will follow a break $(0 \cdot 03)$, while in the $N=1$ case this would still have a relatively high probability $(0 \cdot 2)$. Looking at the probabilities of sequences of non-breaks, we see differences in the probability of a non-break following two previous non-breaks. As $N$ increases, we see that the probability of long sequences of non-breaks decreases $\left(P\left(\left.N_{i}\right|_{i-1}, P_{i-2}\right)=0.8\right.$ for $N=1,0.76$ for $N=2$ and 0.71 as $N=3$ ). Thus a higher order n-gram helps prevent unrealistically long sequences of just non-breaks or just breaks. The POS sequence model probabilities (not shown here) are associated with each state. All states of the same basic type are the same and so the probability distributions for state $B_{B}$ (a break following a break) are the same as for state $B_{N}$ (a break following a nonbreak). 
The probability we are interested in is $P\left(j_{i}\right)$ given the previous sequence of junctures and the POS sequence at that point. This probability can be rewritten as follows:

$$
P\left(j_{i} \mid C_{i}, J_{i-1}^{N}\right)=P\left(\left(j_{i} \mid J_{i-1}^{N}\right) \mid C_{i}\right)
$$

and using Bayes equation

$$
P\left(j_{i}\left|J_{i-1}^{N}\right| C_{i}\right) \propto P\left(j_{i} \mid J_{i-1}^{N}\right) \cdot P\left(C_{i} \mid\left(j_{i} \mid J_{i-1}^{N}\right)\right)
$$

We make the assumption that the probabilities of all states of a particular juncture type are equal (e.g. $P\left(C_{i} \mid\right.$ break, non-break $)=P\left(C_{i} \mid\right.$ break, break $\left.)\right)$, so

$$
P\left(C_{i} \mid\left(j_{i} \mid J_{i-1}^{N}\right)\right)=P\left(C_{i} \mid j_{i}\right)
$$

and from Equation (5) the probability of a juncture type given the preceding types and POS sequence becomes

$$
\mathrm{P}\left(j_{i}\right) \propto P\left(j_{i} \mid J_{i-1}^{N}\right) \cdot P\left(C_{i} \mid j_{i}\right)
$$

\section{Data and evaluation}

We have used the Spoken English Corpus (Arnfield, 1994) for all the experiments described here. This is a database of spoken British English recorded from BBC Radio 4. It is mostly speech read from scripts in the form of news stories, weather reports etc. This corpus has been labelled with POS information and has been hand labelled with major and minor prosodic phrase breaks. The section of the corpus that we use has 39369 words and contains 7750 breaks (both major and minor). The corpus comprises 40 separate stories, of which 30 were used for training and 10 for testing. This resulted in a training set consisting of 31707 words and 6346 breaks, and a test set of 7662 words and 1404 breaks.

\subsection{Performance criteria}

Performance is assessed with reference to $N$, the total number of junctures in the test set, and $B$, the total number of junctures that are breaks. A deletion error $(D)$ occurs when a break is marked in the reference sentence but not in the test sentence. An insertion error $(I)$ occurs when a break is marked in the test sentence but is not in the reference. A substitution error $(S)$ occurs when a break occurs in the right place but is of the wrong type. This type of error is only relevant when more than one type of break is being recognized. There is no single best way to measure the performance of a phrase break assignment algorithm, and a variety of approaches have been proposed in the literature. We explain these performance measures below

$$
\text { Breaks-correct }=\frac{B-D-S}{B} \times 100 \%
$$




$$
\begin{gathered}
\text { Non-breaks-correct }=\frac{N-I-S}{N} \times 100 \% \\
\text { Junctures-correct }=\frac{N-D-S-I}{N} \times 100 \%
\end{gathered}
$$

False insertions w.r.t. junctures $=\frac{I}{N} \times 100 \%$

False insertions w.r.t. breaks $=\frac{I}{B} \times 100 \%$

The difference between breaks-correct and junctures-correct lies in whether non-breaks are included in the calculation. The junctures-correct score gives credit when both the test and reference sentences have a non-break at the same juncture, while the breaks correct score only looks at junctures with breaks. In our data, the number of nonbreaks outnumbers the number of breaks by a ratio of about $4: 1$, and hence an algorithm that marks everything as non-break will score about $80 \%$ junctures-correct, but $0 \%$ breaks-correct (Wang and Hirschberg (1992) give nearly identical figures for the relative number of non-breaks to breaks.) Because the breaks-correct score is not dependent on the relative distributions of breaks and non-breaks, we regard this as a better indicator of algorithm performance. The assessment of insertions is more troublesome: one can either calculate them as a percentage of the number of breaks in the test set or of the number of junctures, as in Ostendorf and Veilleux (1994). For reasons of readability and succinctness we use only the breaks-correct, junctures-correct and juncture-insertion scores in this paper.

\subsection{Testing methodology}

While some algorithms in this field are portrayed as multi-purpose systems, which could be used in speech synthesis, speech recognition or automatic database tagging (Wang and Hirschberg, 1992; Ostendorf and Veilleux, 1994), our system is designed and optimized solely for use in an unconstrained text-to-speech system. To that end we test the system on the input format it can expect in real use: a continuous stream of ascii characters with no division into words, sentences or other units. The division of the text into such units is performed automatically within the initial text processing modules of the text-to-speech system. The POS tagging is performed automatically, and no acoustic features are used. These testing conditions are as close as one can get to how the system would operate in real use: it is important to bear this in mind when comparing these results to other systems.

\section{Part-of-speech tagging}

We use a standard HMM-based tagging framework as is commonly found in a number of systems (e.g. DeRose, 1988). This model consists of two parts: an n-gram model for 
TABLE I. Comparison of basic models

\begin{tabular}{lccc}
\hline Experiment & Breaks-correct (\%) & Junctures-correct (\%) & Juncture-insertions (\%) \\
\hline Det. P & $54 \cdot 274$ & $90 \cdot 758$ & $0 \cdot 852$ \\
Det. PCF & $84 \cdot 402$ & $71 \cdot 288$ & $31 \cdot 728$ \\
Prob P 1-gram & $54 \cdot 986$ & $91 \cdot 099$ & $0 \cdot 799$ \\
Prob P 6-gram & $58 \cdot 547$ & $88 \cdot 006$ & $5 \cdot 385$ \\
Prob PCF 1-gram & $54 \cdot 886$ & $91 \cdot 099$ & $0 \cdot 799$ \\
Prob PCF 6-gram & $68 \cdot 305$ & $89 \cdot 393$ & $5 \cdot 849$ \\
\hline
\end{tabular}

part-of-speech sequences and a likelihood distribution model of part of speech tags for words. These parts are combined using Bayes Theorem and the Viterbi algorithm is used to find the most probable part-of-speech sequence given a set of words.

Although the Spoken English Corpus is marked with POS tags, this corpus has too few words to train a HMM POS tagger. Instead we used the Penn Treebank (Marcus, Santorini and Marcinkiewicz, 1993) which consists of around 1.2 million words from the Wall Street Journal (WSJ). Apart from size, we do not think that the two corpora are significantly different with respect to POS behaviour. The words in the WSJ data are tagged automatically with subsequent hand correction. Punctuation is reduced to a single tag giving us a base tag-set of $K=37$. A generic, unknown word POS distribution is made from the POS distributions of a set of less frequent words and there is a special distribution for words containing just digits. The tagger correctly tagged $94 \cdot 4 \%$ of the words of an independent test set of 113000 words.

The parameters in our POS sequence model are calculated from POS tag occurrences and it is clear that while the full tag-set may potentially be the most discriminative, it also leads to sparse data problems. A series of experiments found that a tag-set of size 23 was the overall best. The reduction in size can be carried out in two ways, either by mapping the output of the tagger onto a smaller tag-set, or by training the tagger on the smaller set. We found that when we post-mapped the tag-set the performance was $97 \cdot 0 \%$, while training and testing on the reduced set gave a worse figure of $96 \cdot 2 \%$. Hence we always use the full tag-set for POS tagging purposes and reduce the size of the set afterwards.

\section{Part-of-speech sequence models}

This section describes experiments that investigate the effect of varying the parameters relating to Equation (2), namely the size of the POS sequence window, $L$; the number of tags before the juncture, $M$ and the size, $K$ and composition, $V$ of the tag-set. In these experiments, for compactness we use two phrase brake models representing ngrams of order 1 and 6. A 1-gram represents the simplest case, and as explained later the 6-gram is the best performing phrase-break model.

\subsection{Punctuation, content words and function words}

First, we report results from two deterministic algorithms, one that uses punctuation only and another that uses this plus the content/function word distinction. No training is necessary in these cases. Row 1 (Det. P) in Table I shows the results from the 
deterministic punctuation-only algorithm, which simply places a phrase break after every punctuation mark in the text. As one might expect, this shows that punctuation is a very reliable indicator of phrase-break presence. The insertion rate is very low at $0 \cdot 852 \%$ showing that punctuation nearly always implies a phrase break. This algorithm correctly finds about half the breaks and inserts very few false ones.

Row 2 (Det. $P C F$ ) shows the results for when breaks are placed after every punctuation mark and before every function word following a content word. The number of breaks correct increases considerably, but only because of a massive over prediction of break placement. The junctures-correct score and the insertion score are the worst for any of the experiments described here.

Rows 3 (Prob P 1-gram) and 4 (Prob P 6-gram) show the results from our algorithm using a POS sequence model with one tag following and one preceding the juncture ( $L=$ $2, M=1, V=\{$ non-punctuation-word, punctuation $\}$ ). Row 3 shows that the probabilistic punctuation-only algorithm produces very similar results to its deterministic counterpart when a 1-gram phrase break model is used, but that break prediction accuracy increases when a higher order n-gram phrase break model $(N=6)$ is used (row 4). Row 5 (Prob. $P C F$ 1-gram) shows that dividing the non-punctuation class into content words and function words has no significant effect on performance $(L=2, M=1, V=\{$ function, content, punctuation $\}$ ) compared to row 3 . If we look at the relevant POS sequence frequency counts in the training data, we find that there are 1751 non-break instances and 1002 break instances for the content-function sequence. Thus a non-break is always more probable, and given a 1-gram phrase-break model, breaks will never be inserted. However, if we use a higher order phrase-break model, the combined probability will become high enough to make breaks more probable if the distance from the last break is more than a few words. The figures in row 6 (Prob PCF 6-gram) show this effect.

In summary, the deterministic punctuation algorithm is "safe" but massively underpredicts, while the deterministic punctuation/content/function word algorithm massively over-predicts. The probabilistic counterparts only perform acceptably if a high order phrase-break model is used.

\subsection{Larger POS tag-sets}

The content-function word rule was motivated by sentences such as "The tall man | was walking $\mid$ down the street" (fccfccfc) where a break occurs after the first noun phrase. While many verb constructions in English contain auxiliaries (function words), constructions such as the simple past do not, and in sentences such as "The tall man walked down the street" (fccccfc) this rule cannot place a break in the same place. Such cases can only be tackled by using a larger tag-set.

To ensure good performance, the POS sequence models must be trained on a sufficient number of examples. There is an inevitable trade-off between robustness, achieved by having a large number of each word sequence in the training data, and discriminative ability, achieved by allowing a large tag-set that can model individual effects. While a tag-set of $K=3, V=\{$ function content, punctuation $\}$ ) is too small, very large tag-sets prevent estimation as the number of possible types of word sequence is $K^{L}$ (i.e. it grows exponentially on the POS sequence window size). Finding the best possible tag-set (one which genuinely optimizes the performance) is a difficult problem and we have not 


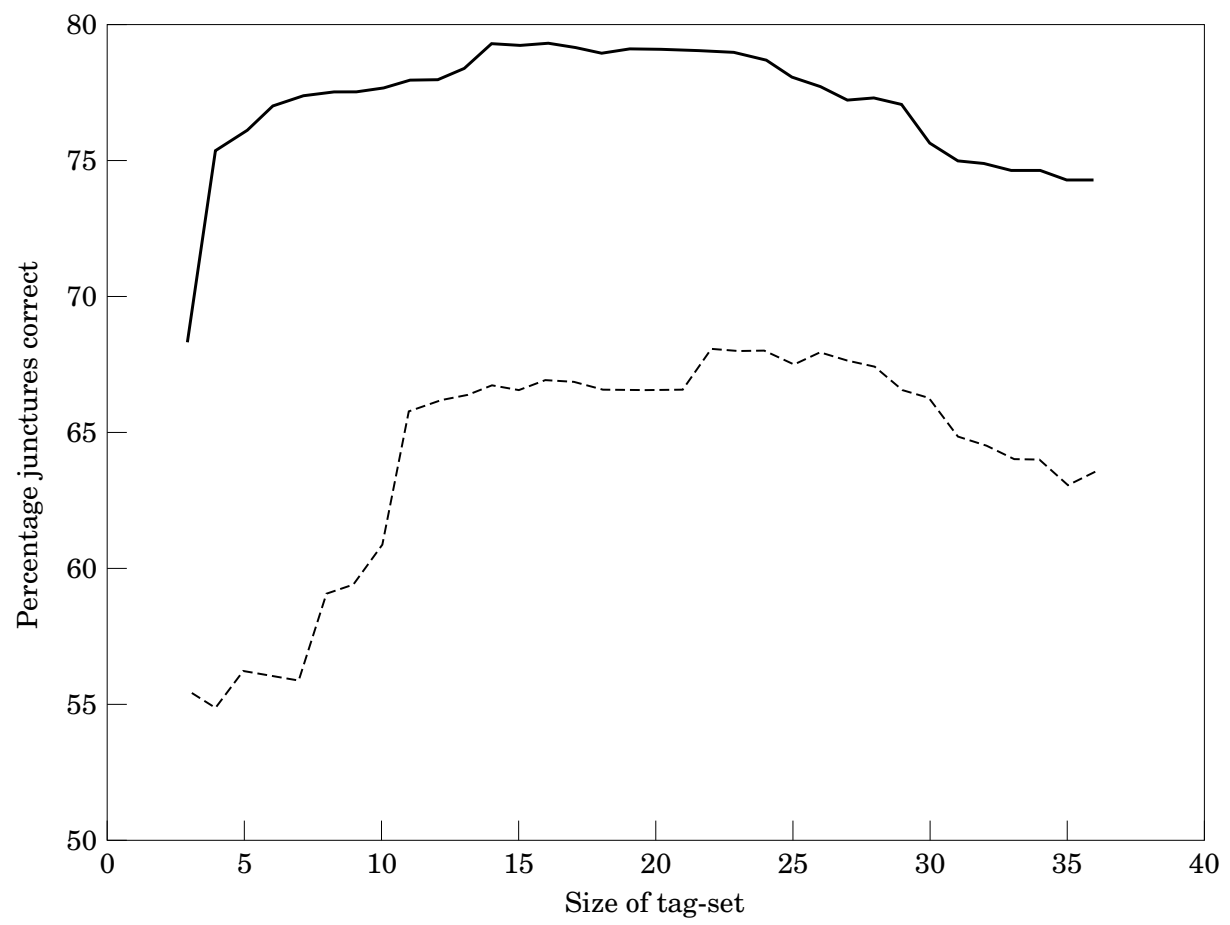

Figure 2. Plot of junctures-correct for a 6-gram (upper line) and 1-gram (lower line) against $K$, the size of the tag-set.

attempted a full solution. Rather we approached the problem empirically and report results from a series of experiments using various tag-sets.

All the experiments here were performed on data tagged with the HMM tagger. It might have been interesting to perform a comparison between the system trained on these tags and the original ones in the corpus that were tagged by hand. Unfortunately the MARSEC corpus has a different tag-set to that of the POS tagger training data and so there was no easy way to perform a rigorous comparison without introducing artifacts from the mapping of one tag-set to the other. However, informal experiments seem to show that the automatic algorithm performs as well (if not better) on automatically tagged data as on hand tagged data.

Figure 2 shows results of an experiment measuring how performance varies as a function of tag-set size. New tag-sets were formed by using a greedy algorithm to collapse categories in the original tag-set. For each stage in the process, we found which combination of two current clusters gave the best performance and chose that as the tag-set for the next stage. We cannot claim that the scores for each tag-set are the best possible for a tag-set of that size as there are many other possible ways to make tagset groups from the original set. However, evidence from this and other experiments conducted on tag-set reduction, where clustering was more linguistically directed, led us to believe that this experiment shows the general trend of performance against tagset size. Again, no single measure can be taken as the sole indicator of performance, but the best results seem to be when the tag-set size is somewhere between 15 and 25 . 
TABLE II. Effect of smoothing on accuracy

\begin{tabular}{lcccc}
\hline Experiment & Phrase-break-model & $\begin{array}{c}\text { Breaks-correct } \\
(\%)\end{array}$ & $\begin{array}{c}\text { Junctures-correct } \\
(\%)\end{array}$ & $\begin{array}{c}\text { Juncture-insertions } \\
(\%)\end{array}$ \\
\hline unsmoothed & 1-gram & $69 \cdot 940$ & $91 \cdot 56$ & 3.600 \\
smoothed & 1-gram & $68 \cdot 376$ & $91 \cdot 46$ & 3.227 \\
unsmoothed & 6-gram & $77 \cdot 070$ & $91 \cdot 49$ & $5 \cdot 270$ \\
smoothed & 6-gram & $79 \cdot 274$ & 91.60 & $5 \cdot 569$ \\
\hline
\end{tabular}

From more detailed analysis involving all the types of phrase models, we found that a tag-set of size 23 gave the best overall performance. This tag-set is linguistically the most easy to describe: the distinctions between subtypes of the four major categories (nouns, verbs, adjectives and adverbs) were ignored, combining the four basic noun tags into a single category and likewise for the six verbial, three adjectival, and four adverbial tags. All punctuation was grouped as one tag. This tag-set of 23 was used in most of the subsequent experiments.

\subsection{Smoothing POS sequence models}

The standard model uses 23 POS tags in a POS sequence model of length 3 . This gives 12167 different possible observations. With a training set of 31707 words it is clear that there will be a large number of POS sequences that never occur or occur only once. In the basic model, sequences with zero counts are assigned a small fixed floor probability. These cases are not particularly important as the chances of breaks and non-breaks being inserted is now governed by the phrase model. More worrying are single occurrences. If a POS sequence is observed only once and with a break at the juncture, this will be assigned the same probability as when a large number of breaks and zero non-breaks are observed for a POS sequence. Clearly the second case is a better indicator that the POS sequence in question really does carry a high likelihood of a break.

To counter this problem we employ a smoothing technique, which adjusts the frequency counts of rare and non-occurring POS sequences. First Good-Turing (explained in Church and Gale (1991)) smoothing is used to adjust the frequency counts of all occurrences for the break and non-break model. This effectively gives zero counts a small value and reduces the counts of rare cases. Next a form of backing-off is applied whereby a juncture likelihood $P\left(\mathrm{c}_{k-1}, c_{k}, c_{k+1} \mid j_{k}\right)$ is discarded if its adjusted frequency count falls below a threshold, and the estimate $P\left(c_{k}, c_{k+1} \mid j_{k}\right)$ is used instead. A threshold of 3 usually gave the best results. Table II gives the results comparing the $V_{23}$ tag-set under smoothing and no smoothing. The smoothed POS sequence models with the 6gram phrase break model are significantly better than the unsmoothed equivalents with both word and break accuracy increasing at only a slight word insertion decrease.

The table shows that smoothing significantly increases performance when used with a high order n-gram phrase break model. 
TABLE III. Results varying the number of words in the POS sequence window and how many are before and after the juncture

\begin{tabular}{lcccc}
\hline Experiment & Phrase-break model & $\begin{array}{c}\text { Breaks-correct } \\
(\%)\end{array}$ & $\begin{array}{c}\text { Junctures-correct } \\
(\%)\end{array}$ & $\begin{array}{c}\text { Juncture-insertions } \\
(\%)\end{array}$ \\
\hline$L=2, M=1$ & 1-gram & $61 \cdot 040$ & $91 \cdot 424$ & $1 \cdot 589$ \\
$L=3, M=2$ & 1-gram & $68 \cdot 376$ & $91 \cdot 464$ & $3 \cdot 227$ \\
$L=4, M=3$ & 1-gram & $61 \cdot 895$ & $90 \cdot 145$ & $3 \cdot 358$ \\
$L=4, M=2$ & 1-gram & $62 \cdot 037$ & $90 \cdot 478$ & $2 \cdot 981$ \\
$L=2, M=1$ & 6-gram & $78 \cdot 134$ & $91 \cdot 104$ & $5 \cdot 913$ \\
$L=3, M=2$ & 6-gram & $79 \cdot 274$ & $91 \cdot 597$ & $5 \cdot 569$ \\
$L=4, M=3$ & 6-gram & $73 \cdot 148$ & $90 \cdot 025$ & $6 \cdot 093$ \\
$L=4, M=2$ & 6-gram & $71 \cdot 937$ & $89 \cdot 786$ & $6 \cdot 110$ \\
\hline
\end{tabular}

TABLE IV. Minor and major phrase breaks

\begin{tabular}{lcccc}
\hline $\begin{array}{l}\text { Phrase-break } \\
\text { Model }\end{array}$ & $\begin{array}{c}\text { Minor correct } \\
(\%)\end{array}$ & $\begin{array}{c}\text { Major correct } \\
(\%)\end{array}$ & $\begin{array}{c}\text { Junctures-correct } \\
(\%)\end{array}$ & $\begin{array}{c}\text { Juncture-insertions } \\
(\%)\end{array}$ \\
\hline 1-gram & $60 \cdot 125$ & $56 \cdot 415$ & $90 \cdot 128$ & $2 \cdot 035$ \\
6-gram & $68 \cdot 703$ & $59 \cdot 502$ & $90 \cdot 211$ & $3 \cdot 923$ \\
\hline
\end{tabular}

\subsection{Varying POS sequence length}

Equation (2) shows the general POS sequence formula, which is expressed in terms of a window of $L$ tags with $M$ of these tags before the juncture and $L-M$ tags after. We can expect longer sequences to be potentially more discriminative, but more prone to sparse data problems. Table III shows results from experiments that varied $L$ and $M$. These were performed on the 23 POS tag-set, using smoothing and a 1-gram and 6gram phrase break model. For both phrase model conditions the $L=3, M=2$ condition outperforms the others.

\subsection{Minor and major}

Most of our work has centred around a model comprising two types of juncture, namely "break" and "non-break", but the algorithm can be easily applied to an arbitrary number of juncture types. The MARSEC corpus is in fact labelled with two types of break, "major" and "minor" and so it was straightforward to build a system that predicted these types. The method is as before, but now three POS sequence models are required and the phrase break model has a vocabulary of three (implying higher perplexities and more sparse data problems). Note that when more than two types of break are used, substitution errors need to be counted for when major breaks are placed where minor breaks occur and vice versa. Table IV reports the results.

We have not yet performed as comprehensive an analysis on multiple break types as with the single type, but have noted that the best tag-sets for the each case are different. While the $V_{23}$ tag-set was the best for the single break case, larger tag-sets gave better results for the multiple case, despite the more severe sparse data problems that occur. 


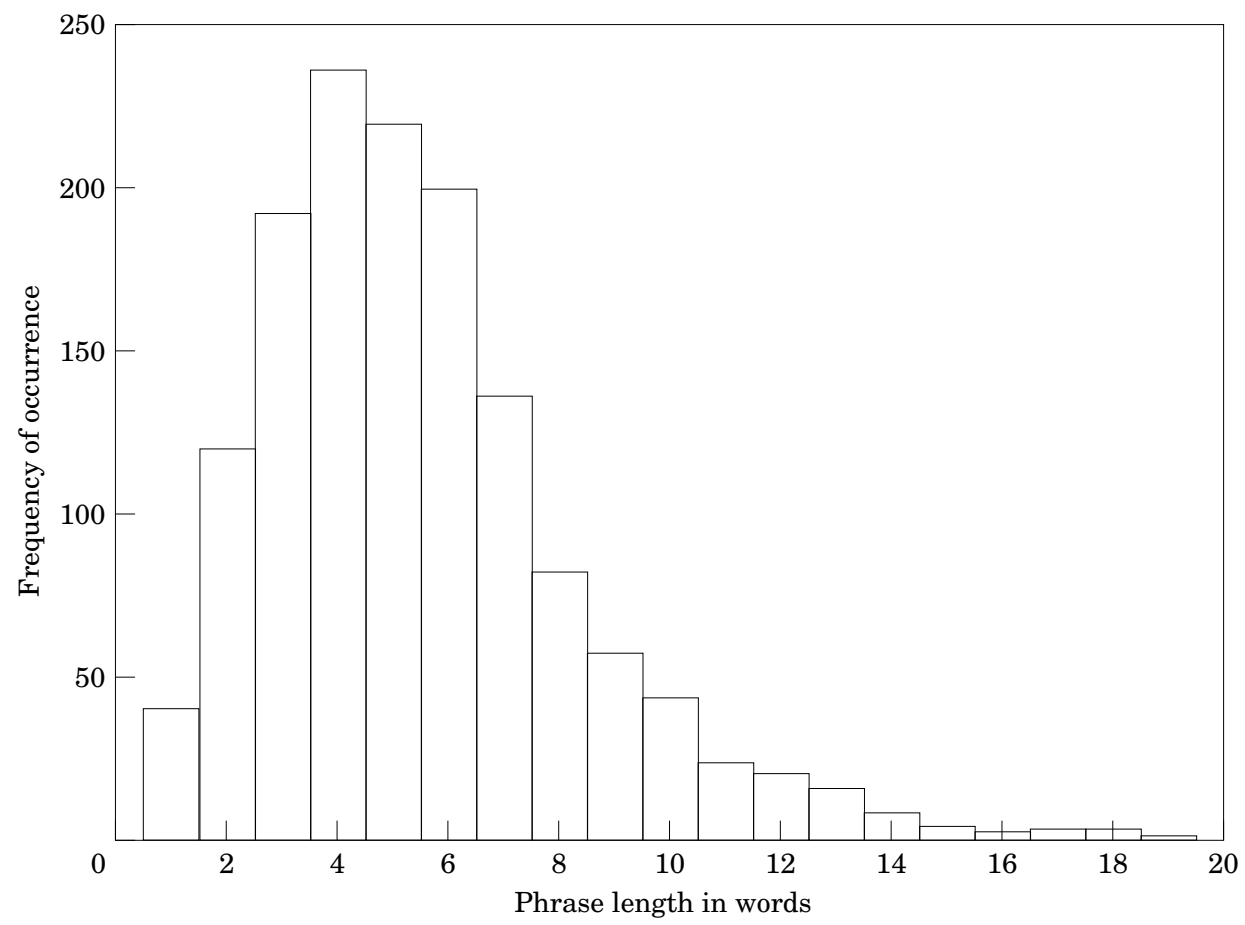

Figure 3. Plot of frequency of occurrence of phrases of different lengths in words.

This indicates that finer distinctions are needed for discrimination of major and minor types.

\section{Using distance information: the phrase break model}

It has been previously noted (Gee and Grosjean, 1983; Bachenko and Fitzpatrick, 1990; Wang and Hirschberg, 1992; Sanders and Taylor, 1995) that phrases occur at somewhat regular intervals and that the likelihood of a break occurring increases with the distance since the last break. The distribution of phrase lengths in words is shown in Fig. 3. It is clear that the majority of phrases are between three and six words long.

Previous work has attempted to use this information (Wang and Hirschberg, 1992; Sanders and Taylor, 1995) but has been hampered by the problem that in a real situation we cannot be certain where the previous break has occurred, and hence it is impossible to reliably compute the distance since the last break. One could simply work in a left to right fashion, placing breaks where they seem most likely and then using that as a definite point to measure the distance to the next hypothesized break. Although simple, this approach is not recommended as a single error cannot be recovered from and may cause errors in all the subsequent decisions. We adopt a different solution, made possible by the use of probabilities throughout the system. Although we cannot say when the previous break to juncture $j_{i}$ occurred, we can estimate the probability that a break occurred at any of the previous junctures $j_{i}-1, \mathrm{j}_{i}-2, j_{i}-3$ and thus try to examine all the possibilities. 
Instead of explicitly modelling the distance from the last break, we use a n-gram model that gives the probability of all sequences of juncture types in a window of size $\mathrm{N}$. (Note this window is completely separate from the window used in the POS model). $\mathrm{N}$-Grams are the most popular choice of language model in speech recognition systems. The number of possible n-grams is given by $T^{N}$, where $T$ is the size of the vocabulary. Because of this, speech recognition language models are usually bigrams or trigrams. Here $T$ is the number of juncture types and in our standard system we have a vocabulary of $T=2$ (break and non-break) and so it is possible to model n-grams as high as 6 and 7 robustly.

The overall goal of the algorithm is to find the most likely sequence of juncture types for the input POS sequence. The probability of a sequence of juncture types $(P(J \mid C))$ is calculated using Bayes' Rule by multiplying the POS sequence likelihood by the phrase probability. In principle, the probability of all possible sequences of juncture types is calculated and the highest is chosen. If calculated directly this entails $S T^{S}$ possibilities where $S$ is the number of words in the sentence. Even for the case when the juncture types are simply break and non-break $(T=2)$, the number of paths can be very large for long sentences (e.g. over a million for a 20 word sentence) and if the $T$ is larger the number of paths becomes intractable. Instead we use the Viterbi algorithm which finds the most likely path in about $T^{2} N$ calculations. For a detailed explanation see Rabiner and Juang (1994).

\subsection{Varying the order of the n-gram}

Perplexity is a measure of average branching factor and can be used to measure how well an n-gram predicts the next juncture type in the test set. If $N$ is the order of the n-gram and $Q$ is the number of junctures in the test set, the perplexity $B$ can be calculated from the entropy $H$ by:

$$
B=2^{H}
$$

where

$$
H=-\frac{1}{Q} \sum_{i=1}^{Q} \log P\left(\left.j_{i}\right|_{j_{i-1}}, j_{i}-2, \ldots ., j i-N+1\right) .
$$

$\mathrm{N}$-grams can be estimated from simple frequency counts of the data. Figure 4 shows how perplexity of a phrase break model of juncture types break and non-break varies as a function of n-gram order. The differences between the various phrase-break models are not large, but it can be seen that the 6-gram has a perplexity of 1.54 compared to the unigram case of about $1 \cdot 62$. It is common in language modelling to use smoothing to account for rare and unseen cases. We recalculated our phrase-break model parameters using three types of smoothing: a fixed floor for unseen cases; Good-Turing smoothing, which alters the probabilities of rarely seen cases as well as unseen cases; and back-off smoothing whereby the values for rare n-grams are computed from the n-1-grams equivalents. None of the types of smoothing had a significant effect on the perplexity 


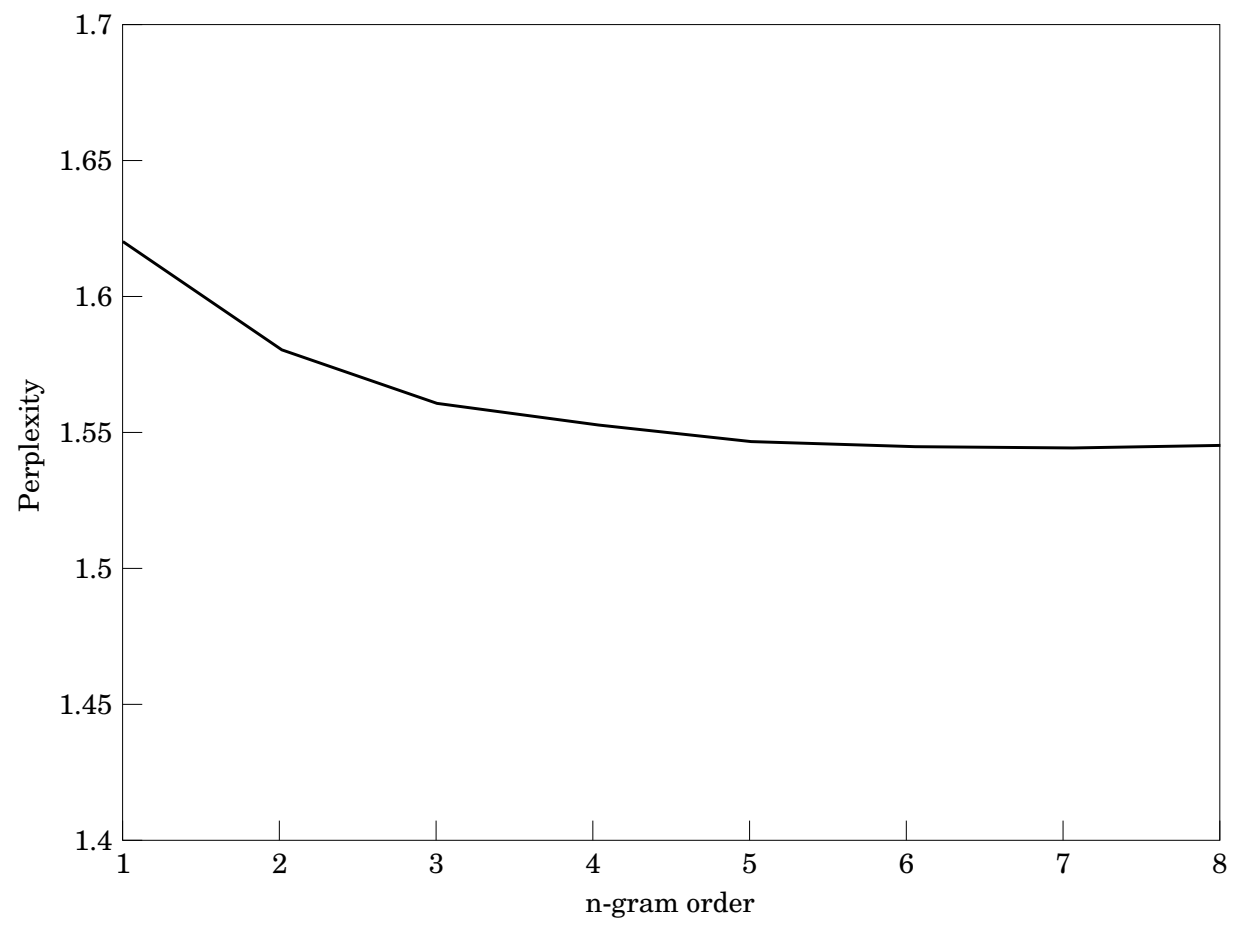

Figure 4. Plot of perplexity against order of n-gram.

or indeed the overall results. In practice we use the simplest type of smoothing where unseen $\mathrm{n}$-grams are given a frequency count of 1 during training.

Figure 5 and Table $\mathrm{V}$ show how the order of the n-gram affects overall performance. The most noticeable effect is the big increse in performance between the unigram phrase-break and the rest, which are fairly similar. This result is due to the phrasebreak model assigning a very low probability $(0 \cdot 03)$ for a break given a preceding break compared with a probability of $0 \cdot 2$ for the same sequence from the unigram. The higher order $\mathrm{n}$-grams perform slightly better than the bigram in terms of junctures-correct and juncture-insertions. In Table V the 7-gram performs the best. In most of our experiments n-grams of order 6 and 7 had consistently better results than the other ngrams, but the difference was often slight. Figure 4 also shows that the perplexity of $\mathrm{n}$-grams of about this order is slightly lower than for the others.

\section{Discussion}

It is worth mentioning a few practical aspects of our algorithm. As the training procedure is fully automated, it is simple to retrain the system on a new prosodically marked database. Training is quick, requiring a single pass over the data for the POS sequence model and the phrase break model. At run-time, the Viterbi decoder requires only a few calculations per input sentence (several orders of magnitude less than the signal processing component of the TTS system, for instance). The framework also allows for some flexibility at run time. As is common with speech recognition Viterbi 


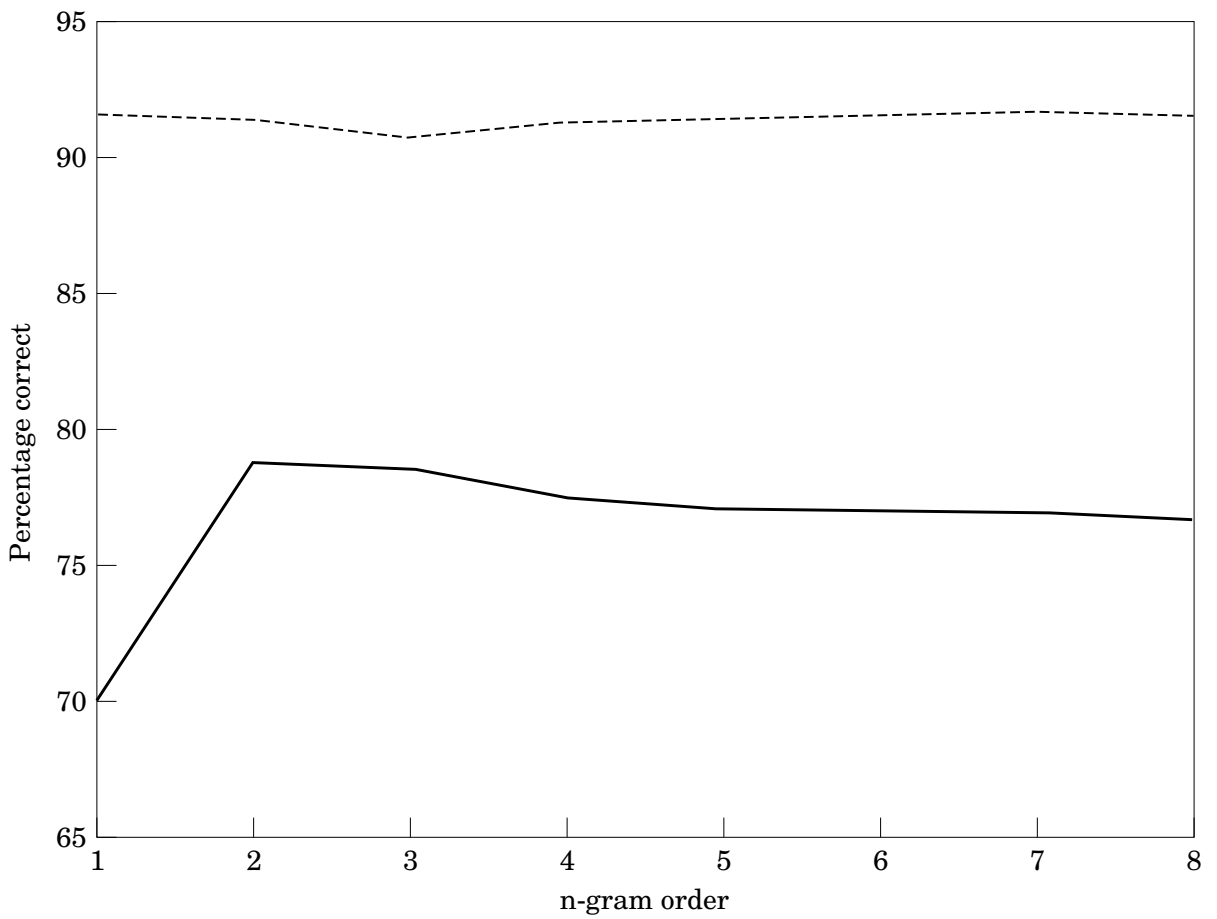

Figure 5. Plot of junctures-correct (upper line) and breaks-correct (lower line) against order of a n-gram.

TABLE V. Comparison of different order phrase break models

\begin{tabular}{lccc}
\hline N-gram order & Breaks-correct & Junctures-correct & Juncture-insertions \\
\hline 1 & $69 \cdot 94$ & $91 \cdot 56$ & $3 \cdot 60$ \\
2 & $78 \cdot 78$ & $91 \cdot 32$ & $5 \cdot 86$ \\
3 & $78 \cdot 56$ & $90 \cdot 67$ & $6 \cdot 62$ \\
4 & $77 \cdot 49$ & $91 \cdot 24$ & $5 \cdot 67$ \\
5 & $77 \cdot 07$ & $91 \cdot 39$ & $5 \cdot 40$ \\
6 & $77 \cdot 07$ & $91 \cdot 49$ & $5 \cdot 27$ \\
7 & $76 \cdot 99$ & $91 \cdot 65$ & $5 \cdot 07$ \\
8 & $76 \cdot 78$ & $91 \cdot 54$ & $5 \cdot 14$ \\
\hline
\end{tabular}

decoders, our system allows a grammar scaling factor that controls the relative importance of the POS sequence and phrase-break models. With some experimentation, this can be effectively used to control the relative insertion and deletion ratios.

The algorithm has been implemented in the Festival speech synthesis system (Black and Taylor, 1997). The setup in Festival uses a POS sequence model based on 23 tags, with two tags before the juncture and one after. Smoothing for the POS sequence model is in the form of Good-Turing smoothing followed by back-off smoothing of threshold 3. An unsmoothed 6-gram phrase break model is used.

We have not yet attempted to use our system on any languages other than English. 
We expect that the algorithm will work with any language that has a phrase structure that can be related to superficial syntactic information such as POS tags.

\subsection{Interpreting results}

Throughout we have given at least three performance figures (junctures-correct, breakscorrect and juncture-insertions) for each experiment. While these gave a reasonably representative indicator of true performance, and have been invaluable in system development, they must be treated with caution. First of all, it is difficult to judge the relative importance of insertion and deletion errors. Our best performing systems often increase the breaks-correct figure to the detriment of the juncture-insertion figure. From listening to the output, we believe that this is an acceptable trade-off, and the best systems from a perceptual point of view are the ones that have the highest breakscorrect while maintaining a high junctures-correct score.

Although formal listening tests might give a better indication of the relative importance of different systems, they are extremely costly compared to the automatic scoring techniques used here. However, it should be noted that a certain amount of "perceptual tuning" can be carried out by scaling the phrase break POS sequence model individually. Thus the relative importance of the two components in Equation (7) can be controlled. The scaling affects the relative numbers of insertions and deletions, and hence the amount of scaling can be set according to which type of error is deemed most significant.

The scoring technique compares the system's placement of phrase breaks with that of a human labeller and so it is important to ask how consistently humans label phrase breaks. Unfortunately, no consistency figures are available for our data, but we think it is safe to assume that the labelling consistency is about the same as that measured by Pitrelli, Beckman and Hirschberg (1994), who obtained a breaks-correct figure of $92.5 \%$ for a similar task. This effectively defines the upper limit one can expect from an automatic system.

It is also important to note that not all errors are of equal importance. Partly this is due to the fact that speakers don't always place breaks in the same place: some junctures can take either breaks or non-breaks without sounding odd, while other junctures must always be of the same type. Ostendorf and Veilleux (1994) proposed a solution to this by having the text in the test set spoken by several different speakers. Sometimes all the speakers agreed, sometimes not. By comparing the output of their system with each instance of the test sentences, it was possible to assess if an error under the usual criterion was actually in a potentially acceptable place. Unfortunately such a comparison measure is not available to us, as we only have a single version of each sentence in the test set.

\subsection{Comparison with other systems}

Our results compare favourably with those of other systems. For example, our best score of breaks-correct is $79 \cdot 27 \%$ compared to $70 \%$ in Ostendorf and Veilleux (1994). Wang and Hirschberg (1992) prefer a measure, which is the average of the breaks correct and the non-breaks correct. Our best score using this measure is $86.6 \%$, which compares with their score of $81.7 \%$ on a text-to-speech task (as opposed to other tasks where they allow acoustic information also). When we ran our system on the test sentences used by Ostendorf and Veileux, we achieved $72 \cdot 72 \%$ breaks correct with 
$4 \cdot 27 \%$ juncture insertions, compared with $70 \%$ and $5 \%$ reported in their paper. This shows that our system has some ability to transfer to completely unseen data in a different domain. Although our system is the best on the common test data, the improvement cannot solely be put down to differences in the techniques themselves. We trained our system on substantially more data and this may have been a large factor in the improvement. Aside from actual performance, we believe our system is somewhat simpler than the two others mentioned here and this may make it more attractive from an implementation point of view.

\subsection{Future improvements}

In this paper we have presented a framework for phrase break assignment from POS information and have attempted a thorough investigation into what the optimal parameter settings in the framework should be. One area we feel still needs further investigation is that of tag-set composition. The experiments in section 5.2 used a greedy algorithm to collapse categories in the original 37 tag-set to form a series of smaller tag-sets. While this is a sensible way to progress we feel that a more sophisticated technique could do better. It may be possible to choose a tag-set based on its actual ability to discriminate juncture types. We have also considered a system where two parallel sets of tags are used, one for before the juncture and the other for after.

While we believe more investigation into tag-set composition would help in reducing errors we also believe that there is only so far that superficial text analysis techniques like this can go. From examining the errors in the test set we believe that a more sophisticated analysis will be needed to correct some of the errors. Although we argued in the introduction against using syntactic parsers for phrase break assignment, our reasons stem from the basic inaccuracy of these parsers, not because syntactic parses themselves are unhelpful. Recently several stochastic parsers have been presented, that are trained on hand parsed trees and employ statistical techniques during parsing, e.g. (Magerman, 1994). These have been shown to significantly outperform rule-driven parsers. It is possible that a statistical parser could provide reliable parses and hence facilitate phrase break assignment.

We would like to thank Steve Isard for his useful comments offered on the draft paper. Special thanks go to Eric Sanders who did much of the early investigative work on POS phrase break detection that lead eventually to the statistical approach developed here. The research reported here was conducted within the Satissfy project, and we gratefully acknowledge the support of the UK Engineering and Physical Science Research Council grant, GR/K45299.

\section{References}

Arnfield, S. (1994). Prosody and Syntax in Corpus Based Analysis of Spoken English. PhD thesis, University of Leeds.

Bachenko, J. and Fitzpatrick, E. (1990). A computational grammar of discourse-neutral prosodic phrasing in English. Computational Linguistics, 16, 155-170.

Black, A. W. and Taylor, P. (1997). The Festival Speech Synthesis System: system documentation. Technical Report HCRC/TR-83, Human Communication Research Centre, University of Edinburgh, Scotland, UK. Available at http://www.cstr.ed.ac.uk/projects/festival.html.

Church, K. W. and Gale, W. A. (1991). A comparison of the enhanced Good-Turing and deleted estimation methods for estimating probabilities of English bigrams. Computer Speech and Language, 5, 19-54.

DeRose, S. (1988). Grammatical category disambiguation by statistical optimization. Computational Linguistics, 14, 31-39. 
Gee, J. P. and Grosjean, F. (1983). Performance structures: A psycholinguistic and linguistic appraisal. Cognitive Psychology, 15, 411-458.

Hirschberg, J. and Preito, P. (1994). Training intonational phrasing rules automatically for English and Spanish text-to-speech. In Second ESCA/IEEE Workshop on Speech Synthesis, Mohonk, New York, pp. $159-162$

Magerman, D. (1994). Natural Language Parsing as Statistical Pattern Recognition. PhD thesis, Stanford University.

Marcus, M., Santorini, B. and Marcinkiewicz, M. (1993). Building a large annotated corpus of English: the Penn treebank. Computational Linguistics, 19, 313-330.

Ostendorf, M. and Veilleux, N. (1994). A hierarchical stochastic model for automatic prediction of prosodic boundary location. Computational Linguistics, 20, pp. 27-55.

Pitrelli, J. F., Beckman, M. E. and Hirschberg, J. (1994). Evaluation of prosodic transcription labelling reliability in the ToBI framework. In ICSLP94, volume 1, pp. 123-126.

Rabiner, L. R. and Juang, B.-H. (1994). Fundamentals of Speech Recognition. Prentice Hall International.

Sanders, E. and Taylor, P. A. (1995). Using statistical models to predict phrase boundaries for speech synthesis. In Proceedings of Eurospeech '95, Madrid, volume 2, pp. 1811-1814.

Silverman, K. (1987). The Structure and Processing of Fundamental Frequency Contours. PhD thesis, University of Cambridge.

Silverman, K., Beckman, M., Pitrelli, J., Ostendorf, M., Wightman, C., Price, P., Pierrehumbert, J. and Hirschberg, J. (1992). ToBI: a standard for labelling English prosody. In Proceedings of ICSLP92, volume 2, Banff, pp. 867-870.

Veilleux, N. M., Ostendorf, M., Price, P. J. and Hufnagel, S. S. (1990). Markov modelling of prosodic phrase structure. In International Conference on Acoustics Speech and Signal Processing, volume 2, 777-780. IEEE.

Wang, M. Q. and Hirschberg, J. (1992). Automatic classification of intonational phrasing boundaries. Computer Speech and Language, 6, 175-196.

(Received 30 June 1997 and accepted for publication 19 January 1998)

\section{Appendix A: Tag-sets}

The full initial 37 tag-set set found from the WSJ is given in Table A.I. The best tagset, shown in Table A.II, is formed by collapsing these tags into 23 tags. As ex, fw and 2 are typically unreliably predicted, and quite rare, they are not included in the implementation released with Festival, and POS tags of this type, if predicted, are treated as part of the nn_nnp_nnps_nns group. Although this marginally reduces the accuracy for our test set it reduces the size of models and hence seems worthwhile in a run-time system. 
Table A.I. The original WSJ tag-set

\begin{tabular}{ll}
\hline Tag & Function \\
\hline CC & coordinating conjunction \\
CD & cardinal number \\
DT & determiner \\
EX & existential "there" \\
FW & foreign word \\
IN & preposition \\
JJ & adjective \\
JJR & adjective, comparative \\
JJS & adjective, superlative \\
MD & modal \\
NN & non-pleural common noun \\
NNP & non-plural proper noun \\
NNPS & plural proper noun \\
NNS & plural common noun \\
of & the word "of" \\
PDT & pre-determiner \\
POS & posessive \\
PRP & pronoun \\
puncf & final punctuation (period, question \\
& mark and exclamation mark) \\
punc & other punction \\
hline RB & adverb \\
RBR & adverb, comparative \\
RBS & adverb, superlative \\
RP & particle \\
TO & the word "to" \\
UH & interjection \\
VB & verb, base form \\
VBD & verb, past tense \\
VBG & verb, gerund or present participle \\
VBN & verb, past participle \\
VBP & verb, non-3rd person \\
VBZ & verb, 3rd person \\
WDT & wh-determiner \\
WP & wh-pronoun \\
WRB & wh-adverb \\
sym & symbol \\
2 & ambiguously labelled \\
& \\
&
\end{tabular}

TABle A.II. The best clustered tag-set. The names directly show the clustering of the original WSJ set

Tag

\section{$\mathrm{cc}$}

$\mathrm{cd}$

$\mathrm{dt}$

ex

fw

in

jj_jjr_jjs

md

nn_nnp_nnps_nns

of

pdt

pos

prp

punc_puncf

rb_rbr_rbs_rp

to

uh

vb_vbd_vbg_vbn_vbp_vbz

wdt

$\mathrm{wp}$

wrb

sym

2 